\title{
Treatment of Immature Scars: Evidence-Based Techniques and Treatments
}

Julian Poetschke and Gerd G. Gauglitz

\section{Contents}

\subsection{Techniques for the Treatment of Immature Scars - 195}

22.1.1 Pressure Therapy - 195

22.1.2 Silicone-Based Products - 197

22.1.3 Onion Extract - 197

22.1.4 Pulsed Dye Laser (PDL) - 198

22.1.5 Fractional Ablative Carbon Dioxide $\left(\mathrm{CO}_{2}\right)$ Laser - 198

22.1.6 Nonablative Erbium Glass (Er:Glass) Laser - 199

22.2 Conclusion $-\mathbf{2 0 0}$

References - 201 


\section{Background}

Effective scar treatment has come a long way in recent years. Classifications allow us to differentiate between individual types of scars and allow us to propose scientifically proven therapeutic regimes to improve even severe pathological scarring. This has helped not only in improving functional and aesthetic results in the affected patients but also to ameliorate their oftentimes severely impaired quality of life.

However, efforts have long been underway to prevent pathological scarring altogether. Hopes are that through early intervention in fresh scars, excessive scarring can be limited or prevented altogether.

The use of compression garments or silicone gel sheeting has long been established to steer the scar maturation process away from excessive and proliferative scar- ring disorders. Flavonoids, too, have proven a promising option for the prevention of excessive scarring in recent years.

Through the advent of newer techniques for the treatment of hypertrophic scars and keloids and fresh insights into the pathophysiology of excessive scarring, new methods for scar prevention are currently making their way into routine practice.

Since fractional photothermolysis has shown its potential in mitigating proliferative scarring and aiding scar remodeling in mature scars, more and more researchers are attempting to alter these ongoing processes in fresh scars to prevent excessive scarring.

This chapter explores the different modalities for scar prevention (see $\bullet$ Table 22.1), their mode of action, and the evidence supporting their use.

Table 22.1 Treatment modalities for immature scars, their indications, and recommendations for treatment

\begin{tabular}{|l|l|l|}
$\begin{array}{l}\text { Treatment } \\
\text { modality }\end{array}$ & Indication & Treatment paradigm \\
\hline
\end{tabular}

Pressure $\quad$ Gold standard for burn injuries after skin grafting therapy or after deeper second-degree burns that have healed without surgery

As an adjunct after earlobe keloid excision

Children with linear hypertrophic scarring or keloids, where intralesional treatment is not an option

Silicone- $\quad$ First-line therapy for linear and widespread based products hypertrophic scarring as well as minor keloids and first option for the prevention of hypertrophic scarring

\section{Onion \\ Can be considered for the treatment of hypertrophic scarring or scar prevention} extract

Pulsed dye

Can be considered for the treatment of laser (PDL)
Begin treatment as soon as the skin grafts are stable enough or after reepithelialization in wounds that have healed without surgery

Garments should be worn for as close to 24 hours per day as possible for 6 to 12 months, depending on the level of scar activity

Garments should be replaced regularly, at least every 3 months so as to ensure sufficiency

Pressure earrings should be worn for as close to 24 hours per day as possible after surgery to minimize the risk of keloid resurgence, starting on the day of suture removal

Same treatment paradigm as in burn scarring. Especially in younger children, sudden growth can require frequent garment replacement

Sheets or patches should be applied to the desired area for 12 to 24 hours per day. Treatment times can vary depending on scar activity, but 3 to 6 months of treatment should be performed

Gels should be applied to the scarred area two to three times per day. They are better suited for mobile areas close to joints

Treatment recommendations vary depending on the manufacturer

Patches are commonly applied for 6 to 12 hours overnight for 3 to 6 months

No clear recommendations or fixed treatment paradigms exist; however, studies indicate that up to three treatment sessions are required for favorable results 
Table 22.1 (Continued)

\begin{tabular}{|c|c|c|}
\hline \multirow{2}{*}{$\begin{array}{l}\text { Treatment } \\
\text { modality }\end{array}$} & Indication & Treatment paradigm \\
\hline & & $\begin{array}{l}\text { In most studies, treatment is begun at the date of } \\
\text { suture removal or shortly thereafter }\end{array}$ \\
\hline \multirow[t]{4}{*}{$\begin{array}{l}\text { Fractional } \\
\text { ablative } \\
\mathrm{CO}_{2} \text { laser }\end{array}$} & $\begin{array}{l}\text { Should be considered in patients where excessive } \\
\text { scarring is expected (after severe burns/trauma) }\end{array}$ & $\begin{array}{l}\text { Expert committees recommend starting treatment one } \\
\text { to three months after the original injury to modulate } \\
\text { the scarring process }\end{array}$ \\
\hline & & $\begin{array}{l}\text { Again, no carefully researched treatment paradigms } \\
\text { exist; however, based on the data available, } \\
\text { low-density, high-energy treatment of the deep dermis } \\
\text { in combination with careful superficial smoothening } \\
\text { seems favorable }\end{array}$ \\
\hline & $\begin{array}{l}\text { Experimentally, the laser has been used } \\
\text { intraoperatively for scar improvement }\end{array}$ & $\begin{array}{l}\text { To aid with the wound healing, this laser has been } \\
\text { used to treat the wound margins intraoperatively } \\
\text { before placing the sutures. One to two passes of } \\
\text { low-density, high-energy laser treatment were } \\
\text { performed on the wound margins }\end{array}$ \\
\hline & & $\begin{array}{l}\text { Thus far, this research was mostly empiric and } \\
\text { uncontrolled; thus, no recommendations regarding its } \\
\text { use can be made, and caution should be used when } \\
\text { considering it }\end{array}$ \\
\hline \multirow[t]{2}{*}{$\begin{array}{l}\text { Fractional } \\
\text { nonablative } \\
\text { Er:Glass } \\
\text { laser }\end{array}$} & $\begin{array}{l}\text { Indications for this laser are similar to those of } \\
\text { the } \mathrm{CO}_{2} \text { laser }\end{array}$ & $\begin{array}{l}\text { Treatment with the Er:Glass laser has less side effects } \\
\text { than with the } \mathrm{CO}_{2} \text { laser; however, its treatment effects } \\
\text { are reduced as well, thus requiring more sessions for } \\
\text { similar results }\end{array}$ \\
\hline & & $\begin{array}{l}\text { As with the } \mathrm{CO}_{2} \text { laser, little research on the potential } \\
\text { for immature scar treatment has been performed, so no } \\
\text { clear recommendations can be made }\end{array}$ \\
\hline
\end{tabular}

\subsection{Techniques for the Treatment of Immature Scars}

\subsubsection{Pressure Therapy}

Upon the discovery of the clinical effects of pressure garments on hypertrophic burn scarring by Silverstein and Larson in the late 1960s, the results of further research on the topic performed at Shriners Burns Institute in Galveston, Texas, were published in the early 1970s. This led to the onset of pressure garment therapy use to prevent hypertrophic scar formation in burn patients. Ever since, this method has become the gold standard for scar prevention in burn patients in many countries around the world. While the exact mode of action has not been fully understood, pressure garment therapy supposedly decreases capillary perfusion in the affected tissue, thus leading to localized tissue hypoxia which in turn increases the rate of fibroblast apoptosis, thus leading to a reduced production of col- lagen. A recent preclinical study by DeBruler et al. investigated the effects of early pressure garment application after skin grafting in burned Duroc pigs and found an increased level of matrix metalloprotease-1 (MMP1) in pigs that received pressure garment treatment within 1 week of grafting [1]. While further research on the matter is required and influences on the levels of collagen I and III or transforming growth factor 1 could not be proven, increased levels of MMP1 which is a known contributor in scar remodeling might help to explain the effects of pressure garment therapy.

Pressure garments come in the form of stockings, pants, sleeves, suits, bandages, girdles, and clip-on earrings, and for locations that are hard to reach through circularly applied pressure, special pelottes can routinely be fitted to allow for effective treatment of nearly every anatomic location. Garments should be worn for as close to 24 hours per day as possible and are usually recommended for at least 6-12 months. In patients showing scar activity after 12 months, treatment is often pro- 
longed for up to 18 months. Being worn for such long times takes a significant toll on the garments which loosen drastically over time, thus requiring regular replacement to ensure sufficient pressure. In our experience, garment replacement is necessary at least every 3 months. To allow patients to wash their compression garments regularly, at least two complete sets should be available at any given time. In patients with physically demanding jobs, wear times might be reduced even further, and more pairs for regular changing might be required.

Pressure garment therapy is very taxing on the patient and compliance is often lacking. Especially during the summer, patients experience sweating and skin maceration, as well as strong odor. In countries without comprehensive health insurance, treatment cost is another important factor. Even in patients where only small areas of the body are affected, treatment over the course of a year will easily cost between one and two thousand euros for a small pressure garment like a glove (- Fig. 22.1) or a sleeve alone, whereas larger pieces or even suits for the whole body will cost low to medium five-figure sums, especially when combined with other treatment modalities.

The current consensus on the pressure applied through garments recommends between 20 and $25 \mathrm{mmHg}$, as studies have shown a higher efficacy when compared to lower-pressure garments $(10-15 \mathrm{mmHg})$.

Even though pressure garment therapy has now been in clinical use for over 40 years, hard clinical data on its efficacy is largely missing, and the existing literature is quite heterogenous.

Early adopters of the technique like Larson, Kischer, and Tolhurst reported favorable results on the reduction of hypertrophic scar formation. More recently, Van den Kerckhove et al. reported positive effects on scar thick- ness when treating patients with garments with at least $15 \mathrm{mmHg}$ of pressure, and Engrav et al. demonstrated that scars under compression were noticeably softer and thinner in their clinical study in 2010 aided by objective measuring devices. However, a large meta-analysis by Anzarut et al. in 2009 was unable to discern differences in scars treated with pressure garments and untreated scars [2]. In 2013, Atiyeh et al. came to a similar conclusion and questioned the widespread use and recommendation, especially in the light of the often poor patient compliance and the resultant cost-effectiveness of this course of treatment. Especially in burn patients, this course of treatment, however, has become the accepted standard. This makes randomized controlled trials with an untreated patient population nearly impossible on ethical grounds, thus limiting the designs of further studies investigating this treatment method.

Thus far, based upon many different studies indicating the clinical efficacy of pressure garment therapy, current guidelines for the treatment and prevention of pathological scarring recommend its use while noting the less than robust level of evidence.

Furthermore, pressure garment therapy can be considered in children with linear hypertrophic scarring or keloids, where intralesional treatment is abandoned because of side effects or the associated risk of the treatment and pressure garment therapy has empirically shown greater efficacy than in adults.

In patients that received surgical treatment for earlobe keloids, pressure earrings have been shown to greatly reduce the risk of keloid recurrence and are thus regularly recommended as an adjunct. Pressure earrings are readily available from specialized orthopedic technicians, and if keloids are excised elsewhere on the ear, like on the helix, tragus, or other parts of the ear, special splints can be custom-made.
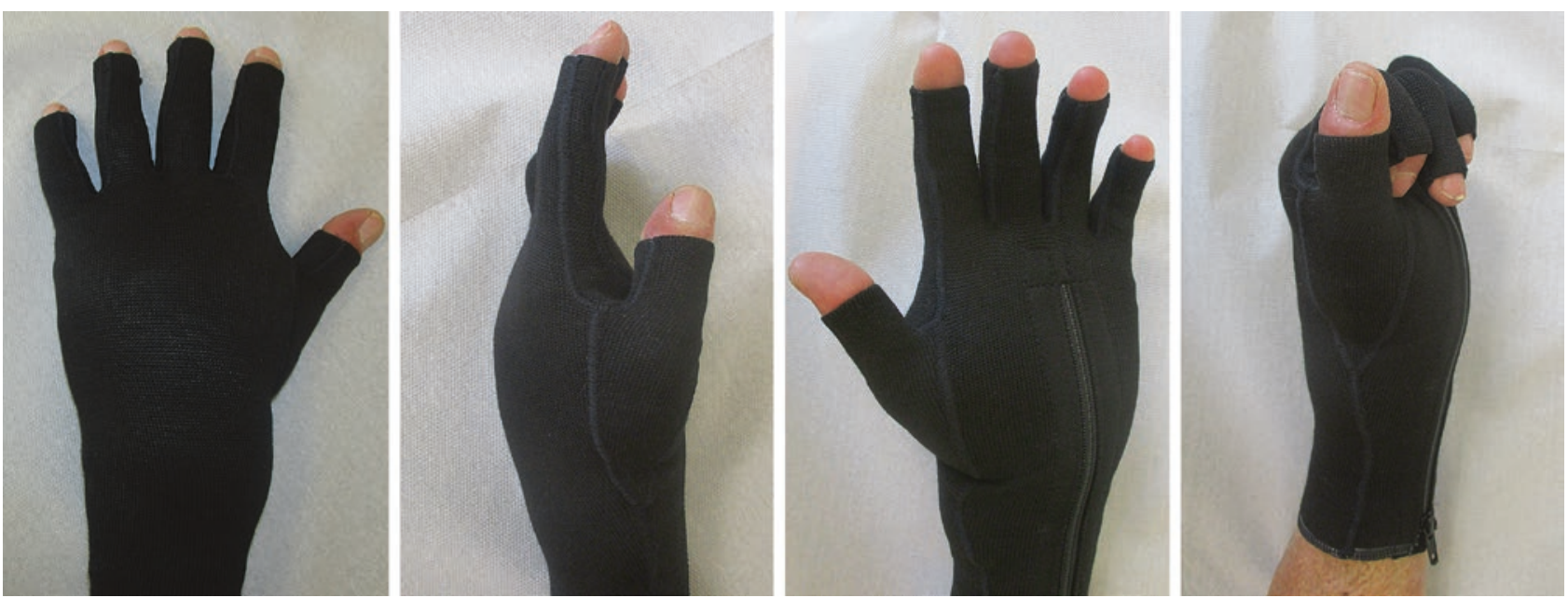

- Fig. 22.1 Patient with severe burn scarring of the left hand wearing a compression glove. The glove allows full function (full finger flexion and extension) while providing full coverage 


\subsubsection{Silicone-Based Products}

Silicone-based products come in a variety of forms. They are available as creams, oils, gels, or, most commonly, patches or sheets that can be applied to the affected areas. Their mode of action regarding the treatment and prevention of excessive scarring has not yet been fully understood, but it is stipulated that the occlusive effect of the silicone inhibits the transepidermal loss of water (TEWL), thus ensuring sufficient hydration of the skin. Thus far, no properties of silicone, which directly influence the process of scarring, have been discovered.

Treatment with silicone-based products can be started after complete reepithelialization of the wounds. Sheets or patches should be applied for 12-24 hours per day throughout 3-6 months. Gel- or cream-based silicone products are better suited for areas that are subject to constant motion (i.e., large joints) where wound dressings might prove unsuitable. To achieve the desired effect, applying the gel two to three times daily is recommended.

Patches and sheets come in many different forms and thicknesses. Commonly, thicker sheets last longer than thinner ones. Larger sheets can be cut into the desired size. They should be cleaned with water and special cleaning solutions that come with the product regularly and can be put back on their carrier foil for storing in between applications. Reusing a single sheet is usually possible for 6-8 weeks, before the silicone disintegrates and becomes gelatinous in its structure, whereupon a fresh sheet should be used.

Side effects or allergies to silicone-based products are rarely observed. Skin maceration through the occlusive effect is possible, whereupon patients can reduce the application time of the silicone.

Silicone has been used in the treatment of immature scars since the early 1980s, where it was first used in the Adelaide Children's Hospital for the treatment of burn scars. Ever since, it has become one of the pillars for early scar intervention. Different prevention and treatment studies have reported outcomes documenting the efficacy of silicone for preventing scar hypertrophy. Cruz-Korchin compared patients after bilateral mammoplasty, after which the scars on one breast were dressed with silicone sheets for 12 hours a day for 2 months while the other remained without preventative treatment. Results demonstrated scar hypertrophy in $60 \%$ of the untreated breasts after 2 months, whereas only $25 \%$ of the scars in the treatment group showed hypertrophy. Ahn et al., in 1991, could demonstrate that surgical incisions treated with silicone gel bandages showed less proclivity for hypertrophy than an untreated control.
In 2001, Gold et al. showed that patients suffering from abnormal scarring showed significantly less hypertrophic scarring when treated with silicone gel sheeting after skin surgery compared to an untreated group of patients.

However, a variety of studies have failed to document significant effects regarding scar prevention of treatment through silicone-based products. In 1998, Niessen et al. failed to show positive effects regarding scar prevention through silicone gel sheeting and even reported a higher rate of hypertrophic scar development than in their control group treated with nonocclusive micropore in their breast reduction scar model.

Cochrane Reviews in 2006 and 2013 noted the generally poor quality of prevention and treatment studies analyzing the effects of silicone regarding pathological scarring [3]. While seemingly reducing the risk of abnormal scarring in high-risk patients and improving scar parameters such as color and softness in existing scars, significant bias was a main critique point in most studies.

Nevertheless, silicone gel sheeting is recommended as a first-line therapy for linear and widespread hypertrophic scarring, as well as minor keloids in national and international guidelines on the management of pathological scarring, and has furthermore been noted as an important option for the prevention of excessive scarring.

\subsubsection{Onion Extract}

Products that contain onion extract have seen increasing popularity for the treatment of immature scars in recent years. They are available as creams or patches and commonly include adjuncts such as allantoin or heparin. Onion extract contains quercetin, a flavonoid that has anti-oxidative and anti-inflammatory properties. Experimental research has suggested that quercetin influences transforming growth factor beta 1 and 2 and SMAD signaling pathways, thus inhibiting fibroblast proliferation and collagen synthesis. In cell cultures, it has also been shown to induce matrix metalloproteinase-1 expression, thus influencing extracellular matrix remodeling.

Scar treatment is begun after complete reepithelialization of the wound is complete. Onion extract patches are commonly used overnight, where they are applied for 6-12 hours over the course of 12-24 weeks, though no clear recommendations regarding the length of treatment exist to date. Gels are often applied multiple times throughout the day for weeks or months, according to manufacturer recommendations, though there is a variance between different formulations and manufacturers. 
Regarding the efficacy of onion-extract-based products, different studies documented significant potential regarding the prevention of pathological scarring. Draelos et al. reported a significantly improved appearance of fresh surgical scars treated with onion extract gel when compared with a control group in two different studies in 2008 and 2012. Parameters positively influenced through the treatment included scar softness, redness, texture, and global appearance. In 2006, Ho et al. evaluated the effects of onion extract gel on scarring after Q-switched Nd:YAG laser tattoo removal in a Chinese patient population and reported significantly less scarring in the treatment group than in the control group.

In 2018, a randomized controlled study by Prager et al. on 125 subjects with fresh postsurgical scars that were treated with an overnight patch containing onion extract, allantoin, and heparin demonstrated significantly better rated scars by both patients and investigators after 12 and 24 weeks of treatment when compared to the control group [4]. Overall treatment comfort was good, and no safety concerns were identified.

Some studies have, however, come up with less positive conclusions. Chung et al. investigated the effects of an onion extract gel when compared with a petrolatum emollient on fresh surgical scars and found no difference between the treatments. Ocampo-Candini et al. published a randomized controlled trial documenting the development of Pfannenstiel scars after cesarean section under treatment with onion extract gel and found no significant improvement over their untreated control group when comparing POSAS scores.

Overall, this relatively new treatment option requires further intense scientific evaluation to improve the level of evidence regarding its efficacy. Due to the promising data that has been collected thus far, current guidelines support the consideration of onion-extract-based formulations for the treatment of hypertrophic scarring as well as the prevention of excessive scarring after surgery.

\subsubsection{Pulsed Dye Laser (PDL)}

The pulsed dye laser is a nonablative laser with a wavelength of 585 or $595 \mathrm{~nm}$. Its target chromophore is oxygenized hemoglobin, and application of the laser will therefore lead to coagulation of capillaries, thus inducing tissue hypoxemia. In scar tissue, this effect will induce a reduction of profibrotic processes and stimulate scar remodeling.

Alster et al. reported significant therapeutic benefits of the PDL for the treatment of keloids in 1995; however, other research groups failed to replicate their findings with some even showing high numbers of recurrence. Further clinical research, however, has established the laser potential for the treatment of erythematous and pruritic scars, for which it is routinely considered. Recently, however, more and more authors have started treating fresh postsurgical scars with the PDL to elucidate whether this can favorably alter the process of scar maturation. McGraw et al. were the first to publish a study on the prevention of hypertrophic scarring with the PDL in 1999. They found that early treatment within the first few weeks after trauma or surgery resulted in a faster resolution of scar stiffness and erythema, as well as a decreased frequency of hypertrophic scarring. Furthermore, they noted an improved scar quality due to the good color match with the healthy skin. In 2003, Nouri et al. published similar findings. In their study, they included 11 patients with 12 postoperative linear scars that were divided into a treated and an untreated half. Overall, three sessions of $585 \mathrm{~nm}$ PDL treatment at monthly intervals were performed, whereupon the cosmetic appearance of the treated scars was reportedly significantly better, when analyzed by a blinded examiner using the Vancouver Scar Scale. In 2006, however, Alam et al. found no significant difference in their randomized controlled trial, where fresh postsurgical scars were treated with one pass of PDL treatment upon suture removal. They noted that 6 weeks after treatment, both groups had improved while the result of neither group was superior. In 2011, Kim et al. examined the effect of three sessions of PDL treatment followed by three sessions of fractional ablative erbium:YAG laser treatment on fresh thyroidectomy scars and noticed favorable effects after PDL treatment with $83 \%$ of patients expressing satisfaction with the result. Er:YAG treatment further improved the observed results [5].

To date, further studies on the potential of the PDL as an option in the treatment of fresh postsurgical scars to quicken scar maturation and to prevent scar hypertrophy exist. However, most studies lack a long-term follow-up that encompasses the entirety of the natural length of scar maturation (12-18 months), thus inhibiting judgment about whether the final results are superior to an untreated control or simply accelerate the process. Therefore, so far, no clear recommendations on the use of the PDL for scar prevention can be made, while the current literature indicates that positive results at the cost of low rates of side effects can be achieved.

\subsubsection{Fractional Ablative Carbon Dioxide $\left(\mathrm{CO}_{2}\right)$ Laser}

While the $10,800 \mathrm{~nm}$ carbon dioxide $\left(\mathrm{CO}_{2}\right)$ laser has been around for decades, the recent development of fractional lasers has led to a significant expansion of its therapeutic range. 
In fractional units, the laser beam is divided into an array of smaller columns, leaving untreated skin islets in between. The divided laser columns ablate tissue and can reach depths of up to $4 \mathrm{~mm}$ newer models, with maybe their most important effects taking place in the dermis. Through the heating of the surrounding tissue, fractional $\mathrm{CO}_{2}$ laser treatment activates heat-shock proteins which in turn stimulate antifibrotic factors such as transforming growth factor beta 3 (TGF- $\beta 3$ ) and matrix metalloproteinases, thus stimulating scar remodeling. Additionally, through classic tissue ablation, scar surface irregularities can effectively be smoothened through use of the $\mathrm{CO}_{2}$ laser.

After the discovery of these effects through experimental research, different clinical studies have since used the fractional $\mathrm{CO}_{2}$ laser to treat mature burn scars. Recent studies found that a single treatment can improve scar softness and surface irregularities by up to $30 \%$ all the while positively influencing patient quality of life. While further research is warranted, current guidelines for the treatment of excessive scarring are recommending the $\mathrm{CO}_{2}$ laser for treatment in severe widespread hypertrophic scarring.

Side effects of the treatment commonly include swelling and oozing of the wounds throughout the first days after treatment, as well as erythema, which commonly recede within 1-2 weeks after treatment. In the author's experience, when treating severe scarring with higher fluences, posttreatment hyperpigmentation often occurs but tapers off after 4-6 months after treatment.

Since the fractional $\mathrm{CO} 2$ laser has seen great success in the treatment of mature widespread scarring and experimental studies suggest that this laser is able to normalize the oftentimes greatly disturbed architecture of the dermal matrix, research is under way to establish the $\mathrm{CO} 2$ laser's abilities regarding excessive scar prevention through the treatment of immature scars.

In 2011, Ozog et al. examined the intraoperative use of the fractional $\mathrm{CO}_{2}$ laser. Before wound closure, onehalf of the wound margins were treated with one to two passes of Active FX (Lumenis Ultrapulse, Santa Clara, California, USA), before the wound was closed. Upon the final evaluation of the scars after 2-3 months post surgery, both patients and examiners rated the lasertreated scar halves superior to the untreated halves [6]. Jung et al., also in 2011, treated immature thyroidectomy scars $2-3$ weeks after surgery with a fractional $\mathrm{CO}_{2}$ laser. Patients were treated with two passes, $50 \mathrm{~mJ}$ of energy and 100 microbeams $/ \mathrm{cm}^{2}$ with a coverage of $12.7 \%$. Analysis of the treatment effect after 3 months revealed significantly improved Vancouver Scar Scale scores, as well as significantly improved values measured with a skin durometer [7]. However, no comparison with an untreated control group was performed. In 2013, Lee et al. performed a split-scar study on 15 three-week-old scars. Two laser passes were delivered at $80 \mathrm{~mJ}$ with a density of 100 microbeams $/ \mathrm{cm}^{2}$ achieving a total coverage of $15.6 \%$. During the final observation, 3 months after treatment, a significant improvement of Vancouver Scar Scale values superior to those made in the control group was found. Especially, scar pliability and thickness improvements were larger than those in the control group, while vascularity and pigmentation did not differ considerably.

Overall, more research on the fractional $\mathrm{CO}_{2}$ laser's abilities regarding scar prevention is required. While initial research shows promising results and the molecular defects thus far discovered suggest that early intervention could lead to a normalization of the dermal architecture in scar tissue, many issues remain to be elucidated. To exclude natural scar regression, which is an important factor in hypertrophic scarring, as a factor, longterm follow-up of over $12-18$ months is required, as well as including a control group.

Nevertheless, expert committees suggest that early treatment, 1-3 months after the original injury, should be considered, especially when excessive scarring is expected. Thus far, no study researching early laser intervention found evidence of increased scarring or other severe side effects, thus supporting the safety the laser is known for in mature scar treatment.

\subsubsection{Nonablative Erbium Glass (Er:Glass) Laser}

The $1550 \mathrm{~nm}$ erbium glass (Er:Glass) laser is a nonablative fractional laser. It does not remove tissue but merely applies heat in a controlled way and leaves the epidermis intact. Through the application of heat in the dermis, its effect there is similar to those of the carbon dioxide laser, albeit at a reduced rate of side effects. Since treatment with the Er:Glass laser does not leave wounds, downtime is greatly reduced. Patients commonly experience light swelling and posttreatment erythema for a few days.

Regarding its efficacy for early scar intervention, thus far, few studies exist. Tierney et al. compared PDL and Er:Glass laser treatment in 2-month-old scars after Mohs surgery in 2009. The study was performed in a split-scar fashion, and patients were blinded as to what side was being treated with which laser. Every scar received four treatment sessions at four-week intervals, and follow-up was performed 1 month after the last treatment session. Overall, patients preferred the results of the Er:Glass-laser-treated scars (83\% of patients). Regarding the scar parameters, pigmentation, scar thickness, and overall aesthetic outcome were all superior in the Er:Glass group [8]. 
In 2014, Ha et al. compared the effects of PDL treatment to Er:Glass laser treatment in a split-scar study on 2-3-week-old thyroidectomy scars. Overall, both groups saw significantly improved scar ratings 6 months after treatment. Patients reported that they found the PDL treated scars less apparent; however, pliability appeared superior in the Er:Glass-laser-treated scars [9]. In their 2014 study, Shin et al. performed another split-scar study, this time comparing ablative fractional $\mathrm{CO}_{2}$ laser treatment to Er:Glass laser treatment. After three treatment sessions in 2-month intervals, follow-up examinations 3 months after the last laser session revealed significant improvement of the scarring in both groups without statistically significant differences between the groups overall. However, the $\mathrm{CO}_{2}$ laser group showed greater improvement of scar hardness, while the Er:Glass-lasertreated scars proved superior regarding their color [10].

Overall, current research has shown promise regarding the Er:Glass laser's efficacy for early scar intervention. However, thus far, few clinical studies are available, thus requiring further research on the matter. As with other laser modalities for the treatment of immature scars, further studies should focus on longer follow-up times as well as larger group samples to further improve the level of evidence.

\subsection{Conclusion}

Efforts to prevent pathological scarring have been underway for many decades. One of the most established methods is pressure garment therapy, which has been used in the prevention of widespread hypertrophic burn scarring since the early 1970s. Years of use have seen this modality become the gold standard, and thus, pressure garments are routinely prescribed for most burn patients in the developed world. However, upon further inspection of the evidence, hard clinical data on the efficacy of pressure garment therapy is only scarcely available. Furthermore, patient discomfort is often high, leading to infrequent treatment compliance. Other options for the treatment of immature scarring are tolerated better. Silicone gel sheeting is often applied throughout the night, and its continued use has been shown to improve scar pliability and height in connection with little to no side effects. Clinical evidence, however, is not as explicit as frequent mentions in national and international guidelines for the treatment and prevention of pathological scarring might infer. Similar in their application to silicone-based products, onion-extract-based gels, creams, and patches have appeared on the market in recent years. Often combined with further active ingredients like allantoin and heparin, they are specifically marketed for use in fresh scars, where they show promise in alleviating scar redness and firmness as well as improving further scar parameters. While additional research will determine the future role of this treatment modality, the low side-effect profile and the appearance of natural ingredients appeal to many patients. In the treatment of mature scars, and increasingly so in the treatment of fresh scars, lasers cannot be ignored. While PDL treatment has been around for years and its use in immature scars to alleviate pruritus and erythema has been well researched and documented, researchers are more and more focusing on fractional lasers. Their effects on dermal matrix remodeling through the stimulation of heat-shock proteins and the resultant effects on transforming growth factor beta isotypes and the concentration of matrix metalloproteases have resulted in researchers trying to employ these effects to modulate the active scar process in favor of remodeling rather than excessive fibrosis. While initial research, both for the ablative $\mathrm{CO}_{2}$ laser and for the nonablative Er:Glass laser, has shown promising results, most recent studies lack sufficient follow-up observation time. To assess whether clinically measured effects are caused through treatment and not through natural scar regression over time, scar maturation must be waited for to make a final conclusion. To date, this presents the largest problems with most research on immature scars. Overall, however, patients today can rely on a variety of promising options for the prevention of excessive scarring. While not every patient requires a robust prevention regimen after surgical intervention, care should be taken to identify patients at risk for excessive scarring and to tailor a treatment algorithm according to their needs. After all, preventing a hypertrophic scar or keloid from forming saves patients from months or years of strenuous symptoms and treatment. Furthermore, today, most algorithms for the prevention of excessive scarring are well tolerated and go along with minimal treatment discomfort and side effects.

\section{Take-Home Messages}

- Pressure garment therapy is the gold standard for the prevention of widespread scarring, e.g., after burns. Even though it has been in use since the early 1970s, evidence for its efficacy is largely empiric. Additionally, side effects of pressure garment therapy often lead to low compliance which affects the treatment efficacy.

- Silicone gel sheeting has proven effective in softening scars, and its use shows little to no side effects. Its mode of action, however, remains largely unknown, and recent meta-analyses question its efficacy. 
- Onion-extract-based products are available as creams and patches. Early research has shown promise regarding the prevention of pathological scarring, but further research is needed to further elucidate its role in the treatment of immature scars.

- PDL treatment has been found to improve pruritus, erythema, and stiffness in fresh postsurgical scars; however, the overall level of evidence for immature scar treatment is low.

- Fractional $\mathrm{CO}_{2}$ laser treatment has been shown to affect dermal matrix remodeling and is therefore increasingly used to modulate immature scars. While initial studies show promise and scar pliability and height seem to improve through treatment, long-term follow-up is crucial in future studies to further strengthen the clinical evidence for this therapeutic option.

- Fractional nonablative Er:Glass laser treatment employs a similar mode of action, much like the $\mathrm{CO}_{2}$ laser, but does not ablate tissue. Thus, downtime and side effects are greatly reduced.

- Scar prevention is imperative in patients with a history of pathological scarring to avoid severe functional, aesthetic, and psychosocial impairments.

- Most modern methods for scar prevention have a minimal likelihood of severe side effects, and treatment discomfort is commonly very low.

\section{References}

1. DeBruler DM, Baumann ME, Blackstone BN, Zbinden JC, McFarland KL, Bailey JK, et al. Role of early application of pressure garments following burn injury and autografting. Plas Reconstr Surg. 2019;143(2):310e-21e.

2. Anzarut A, Olson J, Singh P, Rowe BH, Tredget EE. The effectiveness of pressure garment therapy for the prevention of abnormal scarring after burn injury: a meta-analysis. J Plast Reconstr Aesthet Surg. 2009;62(1):77-84.

3. O'Brien L, Jones DJ. Silicone gel sheeting for preventing and treating hypertrophic and keloid scars. Cochrane Database Syst Rev. 2013(9):Cd003826.

4. Prager W, Gauglitz GG. Effectiveness and safety of an overnight patch containing Allium cepa extract and Allantoin for post-dermatologic surgery scars. Aesthet Plast Surg. 2018;42(4):1144-50.

5. Kim HS, Kim BJ, Lee JY, Kim HO, Park YM. Effect of the 595-nm pulsed dye laser and ablative 2940-nm Er:YAG fractional laser on fresh surgical scars: an uncontrolled pilot study. J Cosmet Laser Ther. 2011;13(4):176-9.

6. Ozog DM, Moy RL. A randomized split-scar study of intraoperative treatment of surgical wound edges to minimize scarring. Arch Dermatol. 2011;147(9):1108-10.

7. Jung JY, Jeong JJ, Roh HJ, Cho SH, Chung KY, Lee WJ, et al. Early postoperative treatment of thyroidectomy scars using a fractional carbon dioxide laser. Dermatol Surg. 2011;37(2):217-23.

8. Tierney E, Mahmoud BH, Srivastava D, Ozog D, Kouba DJ. Treatment of surgical scars with nonablative fractional laser versus pulsed dye laser: a randomized controlled trial. Dermatol Surg. 2009;35(8):1172-80.

9. Ha JM, Kim HS, Cho EB, Park GH, Park EJ, Kim KH, et al. Comparison of the effectiveness of nonablative fractional laser versus pulsed-dye laser in thyroidectomy scar prevention. Ann Dermatol. 2014;26(5):615-20.

10. Shin JU, Gantsetseg D, Jung JY, Jung I, Shin S, Lee JH. Comparison of non-ablative and ablative fractional laser treatments in a postoperative scar study. Lasers Surg Med. 2014;46(10):741-9.

Open Access This chapter is licensed under the terms of the Creative Commons Attribution 4.0 International License (http://creativecommons. $\mathrm{org} /$ licenses/by/4.0/), which permits use, sharing, adaptation, distribution and reproduction in any medium or format, as long as you give appropriate credit to the original author(s) and the source, provide a link to the Creative Commons license and indicate if changes were made.

The images or other third party material in this chapter are included in the chapter's Creative Commons license, unless indicated otherwise in a credit line to the material. If material is not included in the chapter's Creative Commons license and your intended use is not permitted by statutory regulation or exceeds the permitted use, you will need to obtain permission directly from the copyright holder.

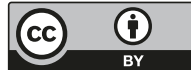

